\title{
Fundamental characteristics of space-charge waves in photorefractive sillenite crystals
}

\author{
Henrik C. Pedersen and D. J. Webb \\ Applied Optics Group, School of Physical Sciences, University of Kent, Canterbury, Kent CT2 7NR, UK
}

Per M. Johansen

Optics and Fluid Dynamics Department, Risb National Laboratory, DK-400o Roskilde, Denmark

Received April 14, 1998; revised manuscript received June 24, 1998

\begin{abstract}
We investigate experimentally the fundamental characteristics of space-charge waves excited in a photorefractive crystal of $\mathrm{Bi}_{12} \mathrm{SiO}_{20}$. Features such as their transient rise and decay as well as their steady-state frequency response are investigated. Based on this, we find the dependence of the space-charge waves' quality factor on spatial frequency and electric-field biasing. The experimental findings are compared with the linear space-charge wave theory developed previously by Sturman et al. [J. Opt. Soc. Am. B 10, 1919 (1993)]. (C) 1998 Optical Society of America [S0740-3224(98)02210-3]

OCIS codes: $190.0190,190.4400,190.5330$.
\end{abstract}

\section{INTRODUCTION}

The concept of space-charge waves is fairly new in the field of photorefractivity. It was introduced by Sturman et $a l .^{1-3}$ and provided a whole new way of describing and understanding the dynamics of space-charge field generation in photorefractive crystals. Unlike earlier presentations ${ }^{4,5}$ the new concept involves a wave equation for the space-charge field from which the characteristics of eigenwaves of the medium in terms of eigenfrequency and eigendamping can be derived.

Apart from putting all the linear processes, such as decay/growth of gratings and running grating generation, into a new perspective, the concept also forms the basis of the nonlinear theory of photorefractive parametric oscillation, of which subharmonic generation is a special case. ${ }^{2,3,6-8}$ One of the main assumptions in this theory is that the quality factor, which is the ratio of the eigenfrequency to the eigendamping, for the space-charge waves is much larger than 1. However, in spite of the large effort in obtaining agreement between theory and experiment, ${ }^{3,9-12}$ only a few investigations of the magnitude of the quality factor of the space-charge waves have been performed. Recently, Lyuksyutov et al. ${ }^{13}$ investigated the propagation of space-charge waves in space, where they screened a part of one recording beam, allowing the traveling space-charge wave to propagate freely into the shadow area. In this experiment they measured a quality factor of more than 100 for the space-charge wave. However, the experiments were performed in a beam coupling geometry. This means that the unscreened recording beam was capable of interacting with its own diffracted beam, which may have helped to lengthen the decay artificially. The process is known as self-enhancement. ${ }^{14}$ In such a case the space-charge wave is not completely free, and consequently one cannot extract its free characteristics, such as eigendamping and eigenfrequency, from the experiment. The concern about the potential influence of beam coupling is reinforced further, partly by the fact that the smallest damping was observed just in the case where the space-charge waves were propagating in the direction known to give maximum beam coupling, and partly by the recent reports on the beam coupling's influence on photorefractive parametric oscillation. ${ }^{15,16}$

In the present paper we investigate the fundamental dynamical characteristics of space-charge waves by using a recording configuration that does not allow beam coupling between the recording beams. It is therefore certain that the space-charge waves are freely propagating and that the observed features reflect the real spacecharge wave behavior. As opposed to the spatial approach adopted in Ref. 13, we use a temporal approach based on well-known decay, ${ }^{17}$ rise ${ }^{18}$ and resonance measurements ${ }^{5}$ in time.

In Section 2 the linear theory for space-charge waves in sillenite crystals is briefly reviewed, and three methods of determining the space-charge waves' quality factor are outlined. These methods are tested experimentally in Section 3, and one is chosen for investigating the quality factor dependencies on grating spacing and applied electric field. The results are discussed in Section 4 .

\section{LINEAR THEORY FOR SPACE-CHARGE WAVES IN SILLENITES}

Our brief review of the linear space-charge wave theory is based on the pioneering formulation by Sturman et al. ${ }^{2,3}$ The principal setup that we have in mind is shown in Fig. 1. Two optical beams, one of which is shifted in angular frequency by $\Omega$, form a moving light intensity pattern in a photorefractive sillenite crystal. The intensity $I$ is assumed to have the form 


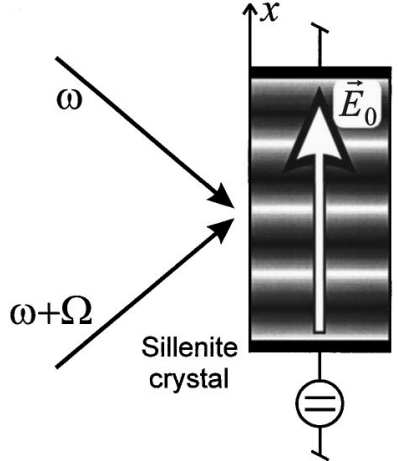

Fig. 1. Schematical setup for the moving grating experiment.

$$
I=I_{0}+I_{1}=I_{0}[1+m \cos (K x-\Omega t)],
$$

where $I_{0,1}$ are the averaged (dc) and modulated (ac) parts of the intensity, $m$ is the modulation coefficient, and $K$ is the magnitude of the intensity grating vector $\mathbf{K}=\hat{x} K$, where $\hat{x}$ is a unit vector along the $x$ axis. Furthermore, a dc electric field $\mathbf{E}_{0}=\hat{x} E_{0}$ is applied to the crystal.

Because the photorefractive crystal is photoconductive, free charges are excited that drift and diffuse to new locations. This causes a charge imbalance in space, but, as the driving intensity pattern is moving, the space charge will have to follow. Hence a space-charge wave is created. From electrostatics (Gauss's law) we know that any charge imbalance creates an electric field; thus the space-charge wave is accompanied by a space-charge field wave that has exactly the same characteristics in terms of eigendamping and eigenfrequency as those of the spacecharge wave. We can therefore choose freely between the two waves in performing the wave characterization. Here we choose the space-charge field.

\section{A. Wave Equation}

Assuming that the intensity modulation is sufficiently small, the space-charge field $E_{1}$ induced in the crystal can be described by the following linearized, one-dimensional wave equation $^{3,6}$ :

$$
\begin{aligned}
& \frac{k_{B} T}{q} \frac{\partial^{2} \dot{E}_{1}}{\partial x^{2}}+E_{0} \frac{\partial \dot{E}_{1}}{\partial x}-\frac{1}{\mu \tau} \dot{E}_{1}+\frac{k_{B} T}{q} \omega_{0} \frac{\partial^{2} E_{1}}{\partial x^{2}} \\
& \quad+\omega_{0} E_{0} \frac{\partial E_{1}}{\partial x}-\zeta I_{0} E_{1}=\zeta I_{1} E_{0}+\frac{k_{B} T}{q} \zeta \frac{\partial I_{1}}{\partial x} .
\end{aligned}
$$

Here $k_{B}$ is the Boltzmann constant, $T$ is the absolute temperature, $q$ is the absolute value of the electronic charge, $\mu$ is the mobility of free electrons, and $\tau$ is the freeelectron lifetime. The parameters $\omega_{0}$ and $\zeta$ are given by $\omega_{0}=s I_{0} N_{D} / N_{A}$ and $\zeta=s q N_{D} / \epsilon_{0} \epsilon_{s}$, where $s$ is the cross section of the photoexcitation of electrons, $N_{D}$ is the density of donors, $N_{A}$ is the density of acceptors, and $\epsilon_{0} \epsilon_{s}$ is the permittivity of the crystal. The dot above some terms in Eq. (2) denotes the time derivative.

Using expression (1) for the intensity and assuming that the space-charge field has the same spatial structure, i.e.,

$$
E_{1}=E_{0} e_{1}(t) \exp (i K x)+\text { c.c. },
$$

where $e_{1}$ is a normalized amplitude and c.c. stands for complex conjugate, one can rewrite the wave equation (2) in the following simple form:

$$
\dot{e}_{1}+\left(\gamma_{K}+i \omega_{K}\right) e_{1}=-i \frac{m}{2} \omega_{K} \exp (-i \Omega t) .
$$

Here $\gamma_{K}$ and $\omega_{K}$ are given by

$$
\begin{aligned}
\gamma_{K} & =\omega_{0}\left(1+\frac{E_{M, K} E_{q, K}}{E_{0}{ }^{2}}+\frac{E_{D, K} E_{q, K}}{E_{0}{ }^{2}}\right), \\
\omega_{K} & =\omega_{0} \frac{E_{q, K}}{E_{0}},
\end{aligned}
$$

where the characteristic photorefractive fields are given by $E_{D, K}=k_{B} T k / q, \quad E_{M, K}=1 / k \mu \tau$, and $E_{q, K}=q N_{A} /$ $k \epsilon_{0} \epsilon_{s}$. In obtaining Eqs. (5), it has been assumed that

$$
E_{q, K} \gg E_{0} \gg E_{D, K}, E_{M, K} .
$$

These inequalities are all fulfilled for the relatively small values of $K$ considered in the present paper.

The physical meanings of $\gamma_{K}$ and $\omega_{K}$ are clear from Eq. (4). As one can see, the equation is identical to that of an ordinary driven oscillator whose damping coefficient and eigenfrequency are given by $\gamma_{K}$ and $\omega_{K}$. Hence, in the present case, $\gamma_{K}$ and $\omega_{K}$ represent the eigendamping and the eigenfrequency of an eigenwave of the space-charge field with wave number $K$. Resonant excitation is obtained when the driving frequency $\Omega$ equals $\omega_{K}$.

An important parameter of the eigenwave is its quality factor $Q_{K}$, given by $\omega_{K} / \gamma_{K}$. It expresses how long the eigenwave survives in free propagation, that is, in the absence of any driving force. Hence $Q_{K} / 2 \pi$ expresses the number of wavelengths that the free wave travels before its amplitude is down to $1 / e$ of its original value. There are several methods of determining the quality factor, of which three are discussed in Subsections 2.B-2.D.

\section{B. Resonance Approach}

The first method is the resonance approach. It is based on the stationary solution to Eq. (4), which is easily found to $\mathrm{be}^{3,5}$

$$
e_{1}=\frac{m}{2} \frac{\omega_{K}}{\Omega-\omega_{K}+i \gamma_{K}} \exp (-i \Omega t) .
$$

Thus, if an ordinary running grating experiment is performed, a space-charge field with the amplitude given in Eq. (7) is generated. If one then performs a passive readout of the phase hologram, formed by means of the photorefractive effect, the following diffraction efficiency $\eta$ is obtained:

$$
\eta \propto\left|e_{1}{ }^{2}\right| \propto \eta^{(\mathrm{i})}=\frac{\gamma_{K}{ }^{2}}{\left(\Omega-\omega_{K}\right)^{2}+\gamma_{K}^{2}},
$$

assuming that $\eta \ll 1$. $\quad \eta^{(\mathrm{i})}$ is here a normalized diffraction efficiency, which equals 1 at resonance. As one can see, a Lorentz-shaped resonance curve is obtained with a maximum at $\Omega=\omega_{K}$ and half-maxima at $\Omega=\omega_{K}$ $\pm \gamma_{K}$. The quality factor can therefore be found by taking the ratio of the resonance frequency to half the full width at half-maximum (FWHM) of the resonance curve. 


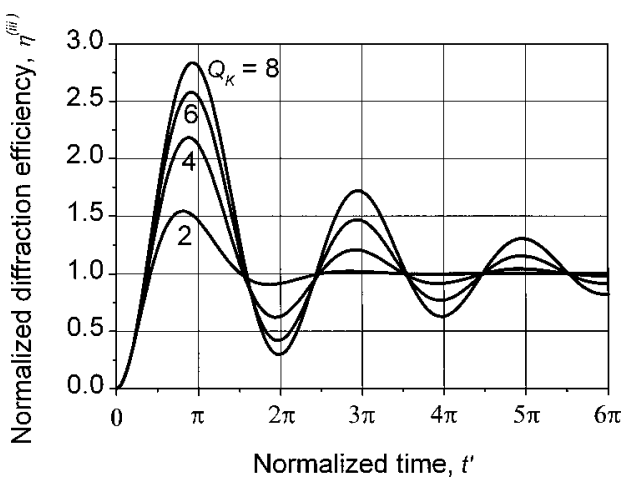

Fig. 2. Transient growth of the diffraction efficiency versus normalized time for four values of $Q_{K}$.

\section{Decay Approach}

Let us assume that the steady state of the space-charge field for some $\Omega$ is reached. If the modulation of the intensity is then turned off (for example, by blocking one of the recording beams), the space-charge field wave becomes free and starts decaying. Solving Eq. (4) for this case (i.e., $m=0$ ), we obtain

$$
\begin{aligned}
e_{1} & =e_{1}(0) \exp \left(-\gamma_{K} t-i \omega_{K} t\right) \Rightarrow \eta \propto \eta^{(i i)} \\
& =\exp \left(-2 \gamma_{K} t\right),
\end{aligned}
$$

where $\eta^{\text {(ii) }}$ is a second normalized diffraction efficiency that equals 1 at $t=0$. Now, by taking $\omega_{K}$ from the resonance experiment and dividing it by the damping coefficient found from the decay experiment, one can obtain the quality factor.

\section{Rise Approach}

In the third method we assume that $\Omega=0$ and that, initially, the intensity modulation is turned off. When it is turned on again, the space-charge field amplitude will start rising. Again, from Eq. (4) we find that

$$
\begin{aligned}
e_{1} & =-\frac{m}{2} \frac{\omega_{K}}{\omega_{K}-i \gamma_{K}}\left[1-\exp \left(-\gamma_{K} t-i \omega_{K} t\right)\right] \Rightarrow \eta \propto \eta^{(\mathrm{iii})} \\
& =\left|1-\exp \left(-Q_{K}{ }^{-1} t^{\prime}-i t^{\prime}\right)\right|^{2},
\end{aligned}
$$

where $\eta^{\text {(iii) }}$ is a third normalized diffraction efficiency, which equals 1 at $t \rightarrow \infty$, and $t^{\prime}$ is a normalized time given by $t^{\prime}=\omega_{K} t$. If we plot the expression for $\eta^{\text {(iii) }}$ versus $t^{\prime}$ for a few typical values of $Q_{K}$, we obtain the curves shown in Fig. 2. As expected, the higher the $Q_{K}$, the more numerous and larger the oscillations that are seen in the diffraction efficiency. We wish to use the height of the first peak, $r$, as a measure of the quality factor. As can be seen in Fig. 2, one can with good approximation say that $r \cong \eta^{(\mathrm{iii})}(\pi)$ for the values of $Q_{K}$ considered here. Thus $r$ can be written as

$$
r \cong\left[1+\exp \left(-\pi / Q_{K}\right)\right]^{2},
$$

which is easily converted to

$$
Q_{K}=-\frac{\pi}{\ln (\sqrt{r}-1)} .
$$

Hence, simply by reading the value of $r$, one can easily find $Q_{K}$ from Eq. (12).
In Section 3 the three methods are applied in some concrete experimental cases.

\section{EXPERIMENTS}

To avoid any influence from beam coupling between the recording beams, all experiments were performed in the non-beam-coupling configuration shown schematically in Fig. 3. This was used for the first time by McClelland et $a l .{ }^{19}$ to prove the existence of subharmonic generation in the absence of beam coupling. The grating recording is performed by two crossed laser beams that propagate at small angles to the $\langle 001\rangle$ crystallographic axis. The formed light interference pattern records a holographic grating with a grating vector directed along the $\langle\overline{1} 10\rangle$ axis, but, because the grating is anisotropic, the recording beams cannot diffract from the grating. Hence no beam coupling takes place between them. To read out the grating, one therefore has to use an additional beam propagating perpendicular to the recording beams, as shown in Fig. 3.

In our case the recording beams were two expanded, linearly polarized beams originating from a diodepumped, frequency-doubled Nd:YAG laser with the wavelength $532 \mathrm{~nm}$. One of the recording beams was reflected off a moving piezomirror driven by a high-voltage controller. Two different signals were used as input to the controller: a low-frequency ramp signal, which effectively shifts the angular frequency of the beam by $\Omega$ and thereby causes the interference pattern to move smoothly along the $\langle 1 \overline{1} 0\rangle$ axis, ${ }^{5}$ and a high-frequency sinusoidal signal with an amplitude that causes the modulation coefficient of the light pattern to vanish. ${ }^{20}$ By switching between these two signals, it was possible to switch the intensity modulation on and off. The recording beam intensity ratio was 1:8000, which corresponds to an intensity modulation of 0.022 , and the total intensity of the two beams was $6 \mathrm{~mW} / \mathrm{cm}^{2}$. The crystal used was a sample of $\mathrm{Bi}_{12} \mathrm{SiO}_{20}$ with the dimensions $13 \mathrm{~mm} \times 13 \mathrm{~mm}$ $\times 6 \mathrm{~mm}$ along the $\langle 001\rangle,\langle 110\rangle$, and $\langle 1 \overline{1} 0\rangle$ crystallographic directions. By means of two electrodes painted on the two (110) crystal faces (see Fig. 3), a dc electric field of up to $15 \mathrm{kV} / \mathrm{cm}$ could be applied. It is worth noting that to avoid electrical breakdown in the air, we kept the electrodes approximately $0.5 \mathrm{~mm}$ from the edges of the crystal.

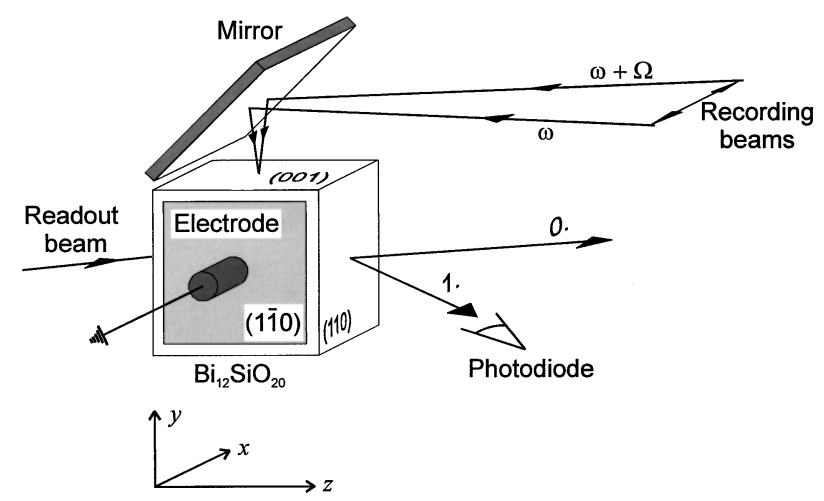

Fig. 3. Schematical diagram of the experimental setup. 
(a)

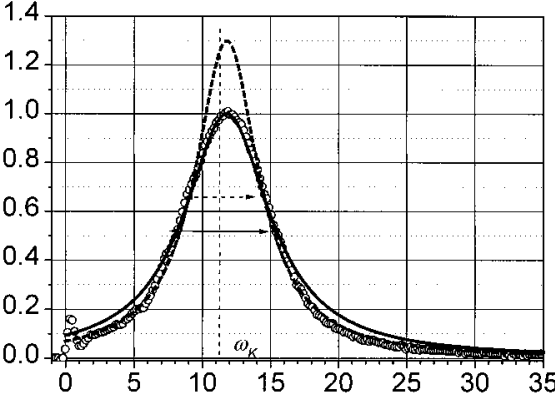

Frequency detuning, $\Omega\left[\mathrm{s}^{-1}\right]$

$$
\begin{aligned}
& \text { s }
\end{aligned}
$$

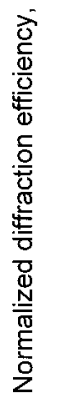

(b)

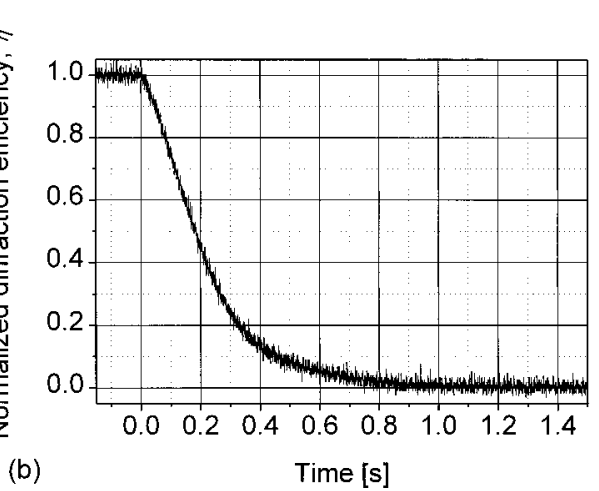

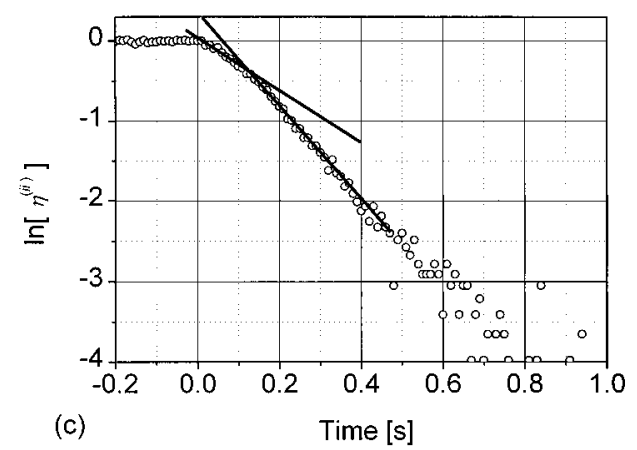

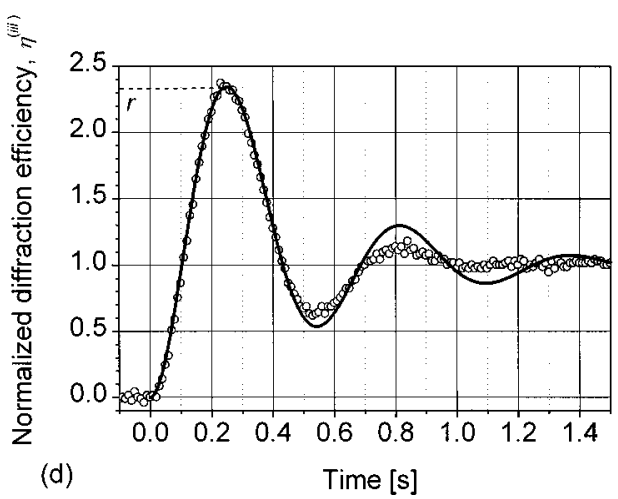

Fig. 4. Normalized diffraction efficiencies measured for $\Lambda=20 \mu \mathrm{m}$ and $E_{0}=8.3 \mathrm{kV} / \mathrm{cm}$. (a) Steady-state, normalized diffraction efficiency versus $\Omega$; the open circles represent measurements, and the curves represent theory. The solid curve is obtained from Eq. (8) with $\gamma_{K}=3.8 \mathrm{~s}^{-1}$ and $\omega_{K}=11.8 \mathrm{~s}^{-1}$; the dashed curve is obtained from Eq. (8) with $\gamma_{K}=2.8 \mathrm{~s}^{-1}, \omega_{K}=11.8 \mathrm{~s}^{-1}$, and a renormalization factor of 1.3. The arrows show the FWHM's of the two theoretical curves. (b) Transient decay of normalized diffraction efficiency at $\Omega=0$. (c) Natural logarithm of the data in (b); the two lines mark two tangents. (d) Transient rise of normalized diffraction efficiency at $\Omega=0$; the open circles represent experiments, and the curve is obtained theoretically from Eq. (10) with $\gamma_{K}=2.4 \mathrm{~s}^{-1}$ and $\omega_{K}$ $=11.3 \mathrm{~s}^{-1}$. The low-frequency peak in (a) is simply a transient peak that occurs when the smooth movement of the piezomirror is turned on, and thus it does not reflect a real peak in frequency response. In obtaining (b) and (d) we switched, at $t=0$, the driving voltage of the piezomirror between a rapid sine voltage and a constant dc voltage.

As a readout beam we used a 1-mW HeNe laser beam, which was always Bragg matched to the grating. The diffraction efficiency was then measured by a photodiode. It was checked that the readout beam did not participate actively in the grating formation/erasure process; hence completely nondestructive readout was obtained.

\section{A. Comparing Methods}

In the first set of experiments, we wished to compare the three methods of measuring $Q_{K}$ outlined in Section 2 . We fixed the grating spacing to $20 \mu \mathrm{m}$ and the applied electric field to $8.3 \mathrm{kV} / \mathrm{cm}$. The results obtained from the three experiments are shown in Fig. 4.

The open circles in Fig. 4(a) were obtained by continuously varying $\Omega$ while measuring the diffraction efficiency $\eta$. This was then normalized to obtain $\eta^{(\mathrm{i})}$, which assumes the value 1 at resonance. The solid curve is obtained from Eq. (8) with $\gamma_{K}=3.8 \mathrm{~s}^{-1}$ and $\omega_{K}$ $=11.8 \mathrm{~s}^{-1}$. These numbers gave the best fit near resonance. At first sight one would say that this curve represents a rather good fit to the experimental points; it would lead to an estimated value of the quality factor of $Q_{K}=11.8 / 3.8=3.1$. However, if one takes a closer look at the solid curve, it may fit well at high values of $\eta^{(\mathrm{i})}$, but at low values, i.e., at each side of the resonance, the fit is actually rather poor, in relative terms. Emphasizing in- stead the fit at each side of the resonance, one ends up with the dashed curve shown in Fig. 4(a). This curve was obtained with the same value of $\omega_{K}$ but with a damping coefficient of only $\gamma_{K}=2.8 \mathrm{~s}^{-1}$ (see the difference in FWHM of the two curves). Moreover, to obtain the best fit, we renormalized by a factor of 1.3. The lower value of $\gamma_{K}$ implies that the quality factor becomes 4.2 instead of 3.1. We will leave the discussion on which value to choose until we know the results of the other methods. Finally, it should be mentioned that by performing several resonance measurements for different values of $\Lambda$ and $E_{0}$, it was established that the resonance frequency was inversely proportional to $E_{0}$ and proportional to $\Lambda$, which is consistent with the linear theory [see Eqs. (5)].

Turning to the decay curves in Figs. 4(b) and 4(c), one immediately realizes a problem: there seem to be two time constants involved, as illustrated by the two tangents in Fig. 4(c). The first part of the decay seems to have a larger time constant than the last part. Repeating the experiment did not necessarily lead to exactly identical results; the time constants usually changed a bit from time to time, as did the apparent number of time constants involved. Thus, unlike the particular case in Figs. 4(b) and 4(c), the first part of the decay could sometimes be faster than the last part. Anyway, by averaging over the time constants, we obtained an eigendamping of 
$\gamma_{K} \cong 2$ to $3 \mathrm{~s}^{-1}$, which, together with the resonance frequency found above, gives a quality factor of $Q_{K} \cong 4$ to 6 .

Finally, turning to the rise curve in Fig. 4(d), one sees that the experimental points fit well to the theoretical curve, as regards the first half of the rise. In the last half the experimental oscillations are smaller than the theory suggests. Thus some extraordinary damping seems to appear at the end of the rise. From the used values $\omega_{K}=11.3 \mathrm{~s}^{-1}$ and $\gamma_{K}=2.4 \mathrm{~s}^{-1}$, which give the best fit, we obtain a quality factor of $Q_{K}=4.7$.

\section{B. Influence of Nonuniform dc Intensity}

A rather obvious question at this stage would be, what is the reason for the discrepancies between theory and experiments shown in Subsection 3.A? The answer, we think, is likely to be nonuniform illumination; that is, an inhomogeneous distribution of the dc intensity $I_{0}$. Strictly speaking, the recording beams do not have uniform cross-sectional distributions of intensity; in fact, the distributions are Gaussian. In our case we found that along the $x$ axis, i.e., in the direction of the applied electric field, the intensity profile was fairly flat because the crystal dimension in this direction is down to $6 \mathrm{~mm}$. However, in the $z$ direction the crystal is $13 \mathrm{~mm}$, and here the intensity varied approximately $\pm 10 \%$, being lowest at the edges. This, of course, causes $\omega_{K}$ and $\gamma_{K}$ to vary along $z$; hence different regions with different $\omega_{K}, \gamma_{K}$ are read out simultaneously. How does this affect the results? To get a rough idea, we consider a simple model to describe the inhomogeneity. This is illustrated in Fig. 5. We assumed that the dc intensity varied stepwise through five steps along the $z$ direction. As a result, five different amplitudes of the running grating would appear in the five slabs. What is the final diffraction efficiency of a beam being diffracted successively in the five slabs? As the model is rather crude already, we may as well take the simplest version of the coupled wave equations for Bragg diffraction in a photorefractive crystal. ${ }^{21}$ If we thus (1) neglect induced birefringence, (2) neglect optical activity, (3) assume that we have exact Bragg readout, and (4) assume that the diffraction efficiency is much less than 1 , we end up with a simple relation for the diffracted beam amplitude $S$, given by $\mathrm{d} S / \mathrm{d} z \propto e_{1}$. Now, as $e_{1}$ varies stepwise along $z$, the final value of $S$ at the exit face of the crystal is found simply by adding up the space-charge fields of each slab weighted by the individual slab thicknesses. Hence the diffraction efficiency, which is proportional to $|S|^{2}$, is simply proportional to

$$
\eta \propto N^{-2}\left|\sum_{p=1}^{N} e_{1}(p)\right|^{2},
$$

where $p$ numerates the layers, $N$ is the total number of equally sized layers, and $e_{1}(p)$ is the space-charge field amplitude in layer $p$.

Let us try to reconstruct the resonance and rise curves in Figs. 4(a) and 4(d) by assuming that $\omega_{K}$ and $\gamma_{K}$ vary in five steps, assuming 90\%, 95\%, 100\%, 105\%, and $110 \%$ of the respective values found in Figs. 4(a) and 4(d). The results of this simple model of varying intensity are shown by the thin dashed curves in Fig. 6. In Fig. 6(a) the effect of the varying intensity is clearly seen to be a reduction of $\eta^{(\mathrm{i})}$ near resonance; in Fig. 6(b) one sees that the effect is a reduction of $\eta^{\text {(iii) }}$ oscillations in the last part of the rise. Hence, in both cases, this was just what we needed to get a better overall agreement between theory and experiment. It therefore seems likely that the non-

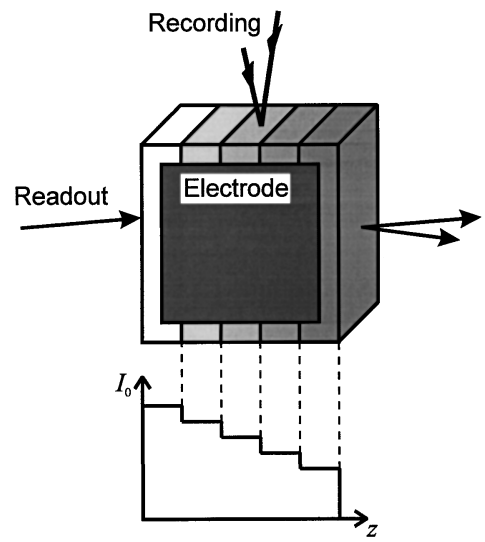

Fig. 5. Schematical illustration of the rough modeling of nonuniform illumination.

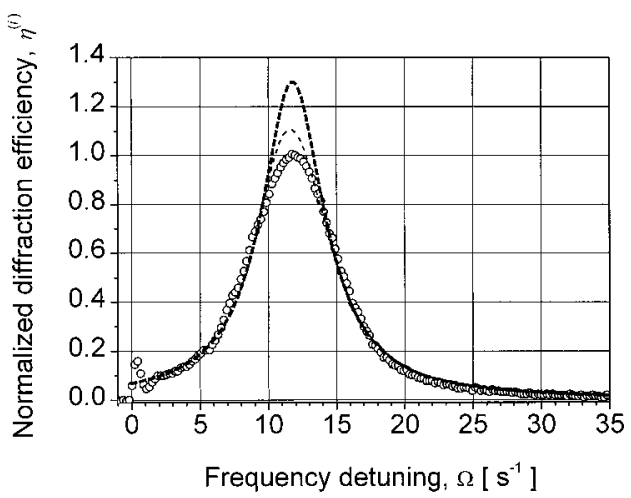

(a)

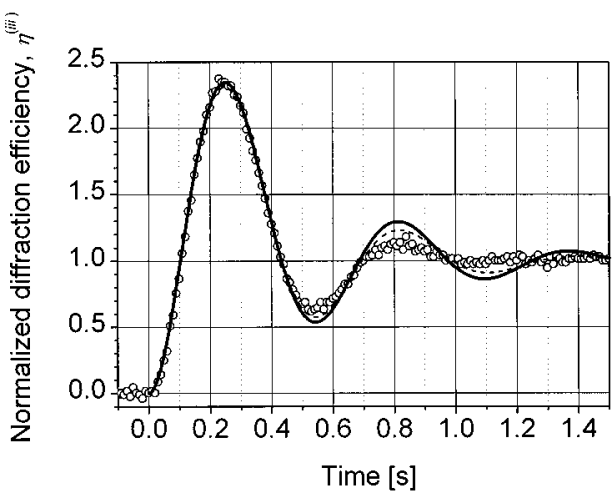

(b)

Fig. 6. (a) New resonance curve (thin dashed curve) obtained from the simple model of including nonuniform illumination. Average values of $\gamma_{K}=2.8 \mathrm{~s}^{-1}$ and $\omega_{K}=11.8 \mathrm{~s}^{-1}$ were used as well as a renormalization factor of 1.3. The thick dashed curve and the circles are taken from Fig. 4(a). (b) New rise curve (thin dashed curve) obtained from the simple model of including nonuniform illumination. Average values of $\gamma_{K}=2.4 \mathrm{~s}^{-1}$ and $\omega_{K}=11.3 \mathrm{~s}^{-1}$ were used. The solid curve and the circles are taken from Fig. 4(d). 

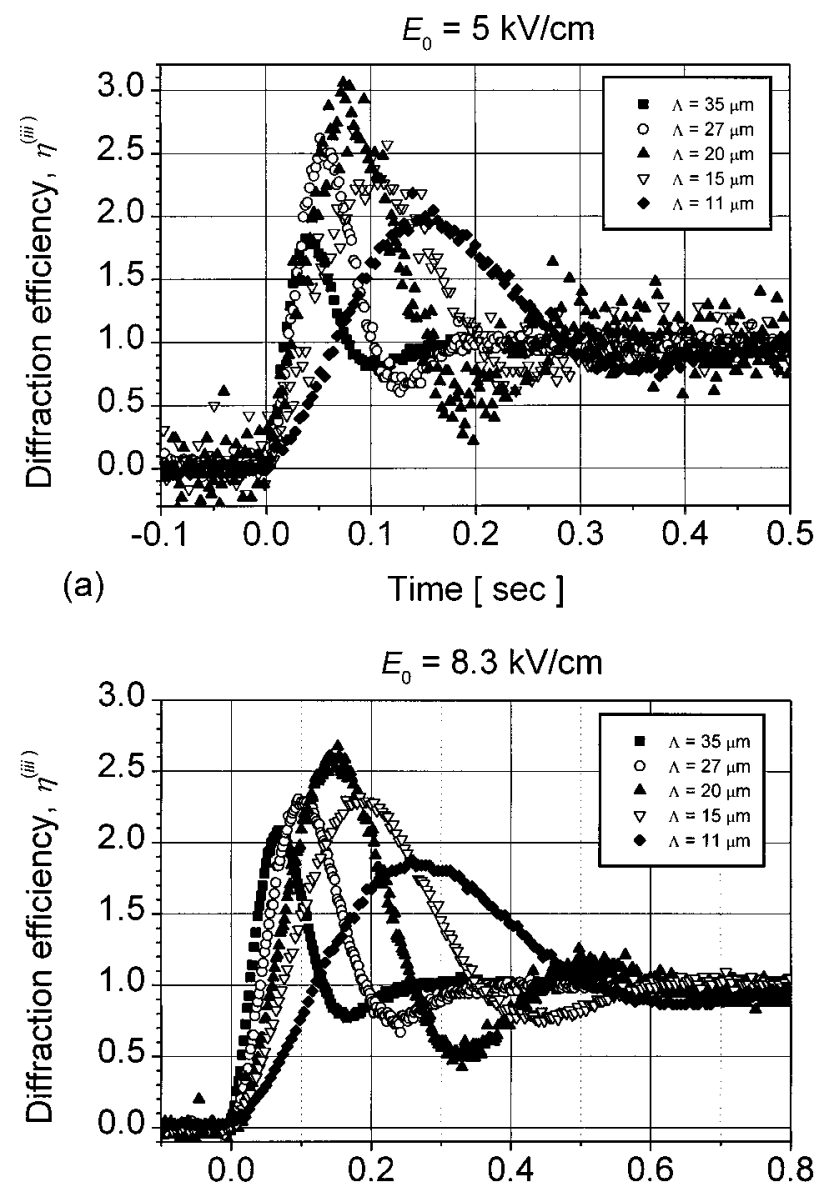

(b)

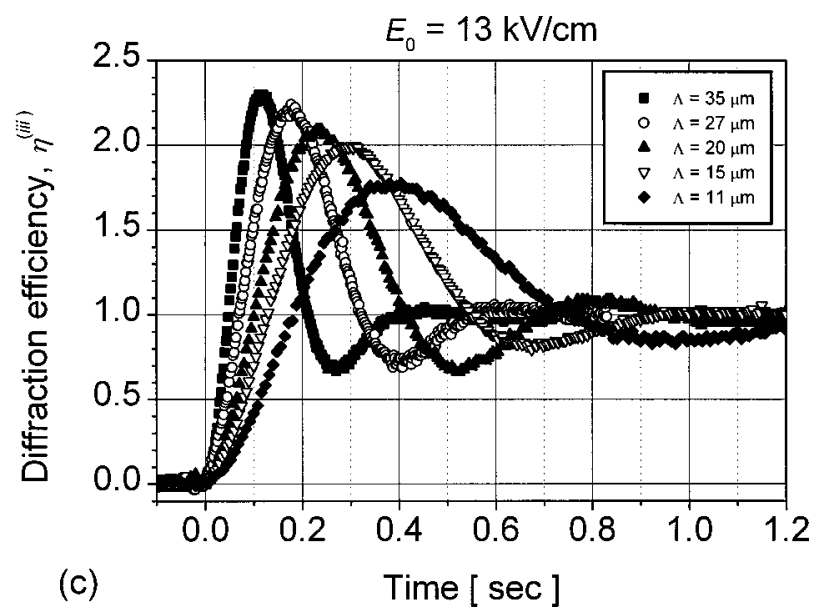

Fig. 7. Rise curves for different values of $\Lambda$ and $E_{0}$.

uniformity of the recording beams is the right explanation for the discrepancies in Fig. 4. Moreover, what is perhaps most important is that this model shows that the varying intensity has a low influence far from resonance and at the first peak of the rise. There should therefore be a good agreement between the $Q_{K}$ obtained from the dashed curve in Fig. 4(a) and the $Q_{K}$ obtained from the $r$ value in Fig. 4(d), since the nonuniform intensity is supposed to have a poor influence on these values. We found the values 4.2 and 4.7 , respectively, which are not too far from each other.
It should be stressed that our simple model of including a nonuniform intensity is far from sufficient for obtaining a complete quantitative agreement with the experiments. It serves only to demonstrate the main effect as well as to show those cases where the nonuniformity has little influence.

Finally, one could ask the question, why not try to expand the recording beams even more and thereby obtain more uniform beams? Our problem with that lies in the fact that our recording laser was not very powerful. Therefore we had to attenuate the readout beam to obtain complete nondestructive readout. Expanding the recording beams even more would force us to attenuate the readout beam further, thereby increasing the noise of the readout process inappropriately.
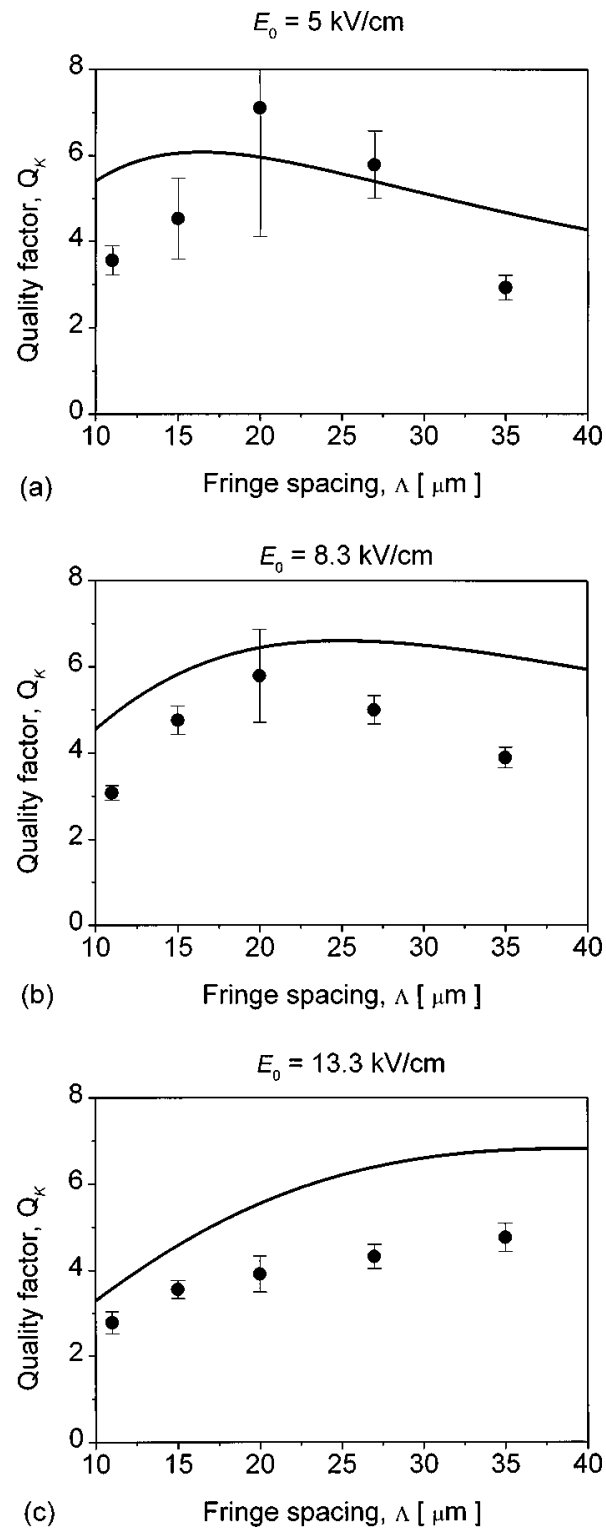

Fig. 8. Quality factor versus fringe spacing for three values of $E_{0}$. The curves are obtained theoretically from Eqs. (5) by using the crystal parameters listed in Table 1 and a value of $\mu$ that was chosen to be $7 \times 10^{-6} \mathrm{~m}^{2} \mathrm{~V}^{-1} \mathrm{~s}^{-1}$ to give the best theoretical fit. The symbols represent experimental values extracted from the $r$ values in Fig. 7. The error bars reflect the uncertainty in determination of the steady-state levels in Fig. 7. 
Table 1. Material Parameters Relevant to $\mathrm{Bi}_{12} \mathrm{SiO}_{20}{ }^{a}$

\begin{tabular}{cc}
\hline$N_{D}$ & $10^{25} \mathrm{~m}^{-3}$ \\
$N_{A}$ & $10^{22} \mathrm{~m}^{-3}$ \\
$\gamma_{R}$ & $1.65 \times 10^{-17} \mathrm{~m}^{3} \mathrm{~s}^{-1}$ \\
$\epsilon_{s}$ & 56 \\
$T$ & $293 \mathrm{~K}$ \\
\hline
\end{tabular}

${ }^{a}$ Ref. 22

\section{Rise Measurements}

In this subsection we wish to investigate the dependencies of the quality factor on fringe spacing and applied electric field with reference to the linear theory. For this purpose we have chosen the rise method, relying on the finding in Subsection 3.B that the first maximum and therefore the $r$ value are not affected by the nonuniform intensity. We performed a series of experiments in which we stepwise changed the angle between the recording beams so that gratings with spacings of $11,15,20,27$, and $35 \mu \mathrm{m}$ were recorded. For every fringe spacing it was ensured that the grating vector was aligned with $\langle 1 \overline{1} 0\rangle$ and that exact Bragg matching of the readout was obtained. Three rise measurements with $E_{0}=5,8.3$, and $13.3 \mathrm{kV} / \mathrm{cm}$ were performed for each spacing. The results are all shown in Fig. 7. As can be seen, the measurements obtained for $E_{0}=5 \mathrm{kV} / \mathrm{cm}$ were quite affected by noise, which caused the determination of the $r$ values, and therefore $Q_{K}$, to become somewhat uncertain. In particular, the uncertainty in steady-state level gives rise to a relatively large uncertainty in $Q_{K}$. However, the tendency for the maximum to be near $\Lambda \cong 15$ to $25 \mu \mathrm{m}$ seems clear, as does the order of magnitude of $r$. The reason for the noise is that a modulation coefficient as small as the one that we used, namely, $m=0.022$, takes a considerable electric field to give a reasonable diffraction signal. That is why the measurements are much clearer for $E_{0}=8.3$ and $13.3 \mathrm{kV} / \mathrm{cm}$.

By using Eq. (12), we extracted the quality factors from Fig. 7. This gave us the $Q_{K}$ dependencies on $\Lambda$ and $E_{0}$ shown in Fig. 8. The agreement with the theoretical curves may be considered fairly good, especially for the high electric fields. There is a reasonable agreement on the order of magnitude of the quality factor, and the observed shift of the optimum fringe spacing toward higher values for increasing $E_{0}$ is consistent with the theory. Moreover, the curve shapes seem to fit quite well, especially at high applied electric fields, because of the reduced noise in the experiments. The fact that the theoretical curves tend to exceed the measured values of $Q_{K}$ as the electric field is increased may be due to electrical screening or contact losses, which reduce the effective internal electric field.

\section{CONCLUSION}

In the present paper we have performed important investigations of the main characteristics of space-charge waves excited in a crystal of $\mathrm{Bi}_{12} \mathrm{SiO}_{20}$. We have briefly described the linear theory of space-charge waves, and three methods of measuring perhaps the most important space-charge wave parameter, namely, the quality factor $Q_{K}$, have been outlined: (1) the resonance approach, based on determining $Q_{K}$ from the steady-state frequency response curve, (2) the decay approach, in which the eigendamping is found from the space-charge wave decay and then divided by the eigenfrequency from the resonance experiment, giving $Q_{K}{ }^{-1}$, and (3) the rise approach, in which $Q_{K}$ is extracted from the rise of the space-charge field. The three methods were tested experimentally, showing some discrepancies with what was expected theoretically. In the resonance curve the experimental measurements showed a reduced space-charge field near resonance, and in the rise curve the experimental oscillations were smaller than the theoretical ones in the last part of the oscillatory rise. The decay approach gave for some unknown reason rather noisy and uncertain results. However, by using a simple model of including the effects of nonhomogeneous recording beams, we have shown that such effects were likely to cause the discrepancies between theory and experiment. So, by choosing certain parts of the experimental curves, in which the inhomogeneous recording intensity had little influence, it was possible to obtain results that were reasonably consistent with theory. Hence we found a good qualitative agreement of the quality factor's dependence on applied electric field and fringe spacing.

As mentioned in Section 1, the quality factor is an important parameter in the theory of subharmonic generation. For the experimental parameter regions considered in the present paper, which are close to the ones used typically in subharmonic generation, the quality factor assumed a value of 4-5. Whether 4-5 is large enough to be considered much larger than 1 , which is one of the basic assumptions in the theory of subharmonic generation, ${ }^{1-3,8,9}$ is hard to tell. At first sight, a quality factor of 4-5 seems to push the theory close to the limit of applicability, but only detailed investigations of each individual case will be able to judge this question accurately. To elucidate this matter, one could suggest analyzing experimentally the dependence of the threshold modulation of subharmonic generation on $E_{0}$ and $\Lambda$. Theoretically, this threshold modulation is found to $b^{3}$ $m_{\text {th }}=3 / Q_{K / 2}$. If this matches the experimental findings, there is a good chance that the theory holds true.

\section{ACKNOWLEDGMENTS}

H. C. Pedersen and P. M. Johansen were supported by the Danish Natural Science Research Council through grants 9502764 and 9600986 . Financial support of the International Association for the promotion of cooperation with scientists from the New Independent States of the former Soviet Union (INTAS project 96-0954) is also gratefully acknowledged.

\section{REFERENCES}

1. B. I. Sturman, M. Mann, and K. H. Ringhofer, "Instability of spatial gratings induced by ac fields in photorefractive crystals," Opt. Lett. 17, 1620-1622 (1992).

2. B. I. Sturman, M. Mann, and K. H. Ringhofer, "Instability 
of moving gratings in photorefractive crystals," Appl. Phys. A: Solids Surf. 55, 235-241 (1992).

3. B. I. Sturman, M. Mann, J. Otten, and K. H. Ringhofer, "Space-charge waves in photorefractive crystals and their parametric excitation," J. Opt. Soc. Am. B 10, 1919-1932 (1993).

4. N. V. Kukhtarev, "Kinetics of hologram recording and erasure in electrooptic crystals," Sov. Tech. Phys. Lett. 2, 438440 (1977).

5. Ph. Refregier, L. Solymar, H. Rajbenbach, and J.-P. Huignard, "Two-beam coupling in photorefractive $\mathrm{Bi}_{12} \mathrm{SiO}_{20}$ crystals with moving grating: theory and experiments," $\mathrm{J}$. Appl. Phys. 58, 45-57 (1985).

6. H. C. Pedersen and P. M. Johansen, "Parametric oscillation in photorefractive media," J. Opt. Soc. Am. B 12, 10651073 (1995).

7. B. I. Sturman, M. Aguilar, F. Agullo-Lopez, and K. H. Ringhofer, "Fundamentals of the nonlinear theory of photorefractive subharmonics," Phys. Rev. E 55, 6072-6083 (1997)

8. E. V. Podivilov, H. C. Pedersen, P. M. Johansen, and B. I Sturman, "Transversal parametric oscillation and its external stability in photorefractive sillenite crystals," Phys. Rev. E 57, 6112-6126 (1998).

9. B. I. Sturman, M. Mann, J. Otten, K. H. Ringhofer, and A. Bledowski, "Subharmonic generation in photorefractive crystals: application of theory to experiment," Appl. Phys. A: Solids Surf. 55, 55-60 (1992).

10. B. I. Sturman, T. E. McClelland, D. J. Webb, E. Shamonina, and K. H. Ringhofer, "Investigation of photorefractive subharmonics in the absence of wave mixing," J. Opt. Soc. Am. B 12, 1621-1627 (1995).

11. H. C. Pedersen and P. M. Johansen, "Longitudinal, degenerate, and transversal parametric oscillation in photorefractive media," Phys. Rev. Lett. 77, 3106-3109 (1996).

12. H. C. Pedersen and P. M. Johansen, "Longitudinal, degenerate, and transversal photorefractive parametric oscilla- tion: theory and experiment," J. Opt. Soc. Am. B 14, 1418-1427 (1997).

13. S. F. Lyuksyutov, P. Buchhave, and M. V. Vasnetsov, "Selfexcitation of space-charge waves," Phys. Rev. Lett. 79 67-70 (1997)

14. J. Otten, A. Ozols, M. Reinfelde, and K. H. Ringhofer, "Selfenhancement in lithium niobate," Opt. Commun. 72, 175 (1989).

15. H. C. Pedersen, P. M. Johansen, and D. J. Webb, "Photorefractive subharmonics: a beam coupling effect?" J. Opt. Soc. Am. B 15, 1528-1532 (1998)

16. H. C. Pedersen, D. J. Webb, and P. M. Johansen, "Influence of beam coupling on photorefractive parametric oscillation in a dc-field-biased $\mathrm{Bi}_{12} \mathrm{SiO}_{20}$ crystal,” J. Opt. Soc. Am. B 15, 2439-2445 (1998).

17. G. Hamel de Montchenault, B. Loiseaux, and J. P. Huignard, "Moving grating during erasure in photorefractive $\mathrm{Bi}_{12} \mathrm{SiO}_{20}$ crystals," Electron. Lett. 22, 1030-1032 (1986).

18. J. M. Heaton and L. Solymar, "Transient effects during dynamic hologram formation in BSO crystals: theory and experiment," IEEE J. Quantum Electron. 24, 558-567 (1988).

19. T. E. McClelland, D. J. Webb, B. I. Sturman, and K. H Ringhofer, "Generation of spatial subharmonic gratings in the absence of photorefractive beam coupling," Phys. Rev. Lett. 73, 3082-3084 (1994).

20. P. M. Johansen, "Enhanced four-wave mixing in photorefractive BSO produced by temporal phase shifts," J. Phys. D 22, 247-253 (1989)

21. A. Marrakchi, R. V. Johnson, and A. R. Tanguay, Jr., "Polarization properties of photorefractive diffraction in electrooptic and optically active sillenite crystals (Bragg regime)," J. Opt. Soc. Am. B 3, 321-336 (1986).

22. P. M. Johansen, "Vectorial solution to the photorefractive band transport model in the spatial and temporal Fourier transformed domain,” IEEE J. Quantum Electron. 25, 530539 (1989). 